\title{
Impact of Oxidative Stress in Fetal Programming
}

\author{
Loren P. Thompson and Yazan Al-Hasan \\ Department of Obstetrics, Gynecology and Reproductive Sciences, University of Maryland School of Medicine, \\ 11-029 Bressler Research Building, 655 W. Baltimore Street, Baltimore, MD 21201, USA
}

Correspondence should be addressed to Loren P. Thompson, lthompson1@umm.edu

Received 5 April 2012; Revised 7 June 2012; Accepted 21 June 2012

Academic Editor: Janna Morrison

Copyright (C) 2012 L. P. Thompson and Y. Al-Hasan. This is an open access article distributed under the Creative Commons Attribution License, which permits unrestricted use, distribution, and reproduction in any medium, provided the original work is properly cited.

\begin{abstract}
Intrauterine stress induces increased risk of adult disease through fetal programming mechanisms. Oxidative stress can be generated by several conditions, such as, prenatal hypoxia, maternal under- and overnutrition, and excessive glucocorticoid exposure. The role of oxidant molecules as signaling factors in fetal programming via epigenetic mechanisms is discussed. By linking oxidative stress with dysregulation of specific target genes, we may be able to develop therapeutic strategies that protect against organ dysfunction in the programmed offspring.
\end{abstract}

\section{Introduction}

This paper addresses the emerging role of oxidant molecules in fetal programming. Oxidative stress is associated with the generation of reactive oxygen species (ROS), which have both physiologic and pathologic roles in the placenta [1], embryo [2], and the fetus [3-5]. We will address recent reviews and current studies that present evidence identifying the impact of oxidative stress on the fetus and how it may contribute to the permanent alterations in the offspring through programming mechanisms.

\section{Intrauterine Stress and Fetal Programming}

Epidemiological evidence and animal model studies have identified a clear association between low birth weight and an increased incidence of hypertension, type II diabetes, metabolic syndrome, insulin resistance, and obesity [6-9]. This was first reported by Dr. David Barker in relation to the inverse relationship between birth weight and mortality rate due to cardiovascular disease [10-12] and systolic blood pressure [13]. Subsequently, babies of higher than normal birth weight were shown to have increased risk of metabolic disorders as adults [14, 15]. Extensive study has lead to identifying additional factors that include altered nutrition, glucocorticoid exposure, and prenatal hypoxia as intrauterine stressors initiating fetal programming $[8,9]$.

The relationship between adult disease (e.g., hypertension, insulin resistance, diabetes) and birth weight forms a U-shape curve demonstrating increased risk with both low [10-12] and high birth weight $[16,17]$. The impact of intrauterine stress on the affected offspring is influenced by the severity and duration of the insult, as well as, the gestational age of fetal exposure $[8,9]$. In conditions of maternal undernutrition, the fetus adapts by reducing its metabolic energy supply to protect critical organs for survival, first described as the Thrifty Phenotype Hypothesis [18]. With further study, the Predictive Adaptive Hypothesis was proposed to describe the fetal response to an anticipated undernourished postnatal environment [19]. Overnutrition (i.e., high fat and carbohydrate diet) during pregnancy also imposes an intrauterine challenge because the fetus is unable to properly regulate its nutrient excess, resulting in a greater than normal birth weight [20]. This is likely due to an imbalance of the appropriate complement of nutrients required for proper development of the fetal organs $[9,21]$. Thus, high or low birth weight, resulting from altered growth 
patterns during intrauterine stress, carries an increased risk of adult disease for the offspring [6-9].

\section{Role of Oxidative Stress in Normal Fetal Development}

The development of the embryo occurs in a relatively lowoxygen environment. It is highly sensitive to injury to oxidant molecules because of its low antioxidant capacity $[22,23]$. As placentation progresses, there is increased oxygen transfer, which increases the cellular generation of ROS [1]. This initiates a switch in the cellular redox state, from reduced to oxidized, acting as a driving force for cell differentiation $[23,24]$. ROS serve as signaling molecules that induce transcription of several genes (e.g. HIF1A, CREB1, NFKB1) important in oxygen sensing, cell differentiation, and proliferation $[1,24,25]$. With placental maturation, there is increased oxygen transfer to the developing fetus, which is required for sustaining the increased metabolic rate during the rapid fetal growth phase [2]. While both nutrition and oxygenation are important for fetal growth, identifying the specific effect of hypoxia in the absence of altered nutrition is important for understanding the role of hypoxia in fetal programming. Recent study has identified the effect of reduced oxygenation, independent of nutrition, using a chick embryo model [26]. Incubation of fertilized eggs from hens at high-altitude resulted in reduced fetal growth compared to eggs obtained from hens at sea level. The restricted fetal growth occurring at highaltitude was corrected by incubating fertilized eggs from high-altitude hens at sea level or by oxygen supplementation that matched sea level conditions. Thus, in the presence of adequate nutrition, reduced oxygenation can negatively impact normal growth and development.

Recent animal studies have identified an important role of oxidant molecules in fetal cardiovascular function under normal conditions [3-5]. Antioxidant administration of melatonin or vitamin $\mathrm{C}$ to pregnant sheep increased umbilical vascular [5] and fetal femoral artery conductance [4], respectively, by mechanisms associated with increased nitric oxide (NO) bioavailability following decreased superoxide anion levels. In addition, inhibition of superoxide anion generation by allopurinol, a xanthine oxidase inhibitor, attenuated the fetal sheep pressor responses to phenylephrine and enhanced umbilical vascular conductance by altering NO bioavailability and activation of $\beta 1$ adrenoceptor mechanisms, respectively [3]. Thus, oxidant molecules play an important physiological role in normal fetal cardiovascular homeostasis. A delicate balance may exist between optimal oxygen levels and the generation of ROS molecules during gestational periods of embryonic and fetal development.

\section{Intrauterine Stress and Hypoxia}

Fetal hypoxia is one of the most significant challenges facing obstetric clinical practice. Intrauterine stress can be generated under conditions of placental insufficiency [27], preeclampsia [28], high-altitude environments [29-33], and exposure to toxic substances [34]. High-altitude living exposes populations to hypobaric hypoxia and imposes a significant challenge to the pregnant woman and her developing fetus. Low birth weight and preeclampsia are common characteristics of high-altitude pregnancies [29-33]. The risk of small size at birth is associated with the increased incidence of high blood pressure and heart disease in the adult offspring [31]. While preeclampsia is associated with oxidative stress [28], it is unclear whether oxidative stress contributes to pregnancy complications that occur at highaltitude.

Animal studies have shown that intrauterine hypoxia induces fetal growth restriction [26, 35], cardiovascular dysfunction $[36,37]$, and multiorgan morbidities of the fetus associated with brain [38-40], heart [41-44], liver [4547], and kidney [7, 48]. Further, recent studies have linked prenatal hypoxia to an increased risk of cardiac $[4,5,36,46$, $49,50]$, vascular [51-53], and metabolic [46] dysfunction in the offspring. Thus, the condition of intrauterine hypoxia impacts the fetus on a multiorgan level and increases the risk of adult disease via mechanisms associated with fetal programming $[8,54]$.

Understanding the impact of intrauterine hypoxia and its generation of ROS during gestation is important for understanding the consequences of both fetal and neonatal outcomes. The effect of ROS on both genetic and epigenetic mechanisms is implicated in the underlying cause of fetal programming although further study is needed. Understanding the contribution of ROS in fetal programming will greatly improve our understanding of the impact of oxidative stress on the long-term consequences contributing to organ injury of programmed offspring.

\section{Generation of Oxidative Stress}

Oxidative stress occurs when the generation of reactive oxidant molecules exceeds the capacity of the cell's antioxidant defense mechanisms. Both enzymatic (i.e., MnSOD, CuZnSOD, Catalase) and nonenzymatic (GSH/GSSG, peroxiredoxin, thioredoxin, ascorbic acid or Vitamin C, tocopherol or Vitamin E) antioxidant pathways are present to combat excessive ROS generation. The major ROS molecules include superoxide anion $\left(\mathrm{O}_{2}^{-}\right)$, hydroxyl radical $(\bullet \mathrm{OH})$, and hydrogen peroxide $\left(\mathrm{H}_{2} \mathrm{O}_{2}\right)$, as well as, others generated by their interaction with reactive molecules, such as, NO and peroxynitrite [55]. Superoxide anions are generated by plasmalemmal- and mitochondrial-associated NADPH oxidases, xanthine oxidase, mitochondrial respiration and by products of several metabolic pathways, such as, fatty acid oxidation [55]. The predominant generators of ROS are cell type specific and their formation is dependent on the cell's metabolic energetics $[56,57]$. Mitochondria are considered primary generators of $\mathrm{O}_{2}^{-}$under conditions of reduced oxygen $[55,56]$ although xanthine oxidase has been shown to contribute significantly to cardiovascular homeostasis in fetal sheep [3-5]. Under normal oxygen conditions, the electron transport chain leaks $\mathrm{O}_{2}^{-}$from Complexes I and III, 
which are normally balanced by mitochondrial SOD (i.e., MnSOD) [57]. With hypoxia, a reduction of molecular $\mathrm{O}_{2}$, as the electron acceptor at terminal complex IV, can result in leakage of electrons and generation of $\mathrm{O}_{2}^{-}$leading to oxidative stress [57].

Targets of oxidative stress include phospholipid membranes, proteins, and nucleic acids [58]. Plasma and organelle membranes are particularly vulnerable to oxidative stress because phospholipids are easily oxidized by $\mathrm{O}_{2}^{-}$. ROS molecules can directly interact with both proteins and DNA to oxidize aminoacids, which disrupt normal structure and function. In addition, mitochondria are predominant targets of oxidative stress because the mitochondrial genome lacks histones and has fewer DNA repair mechanisms than the nucleus [59]. Damage to mitochondrial proteins and membranes can lead to further mitochondrial dysfunction. This can result in a positive feedback mechanism by which failure of only a few mitochondria, injured by oxidative stress, can recruit an entire network of mitochondria to fail [60]. Thus, organs that have a high reliance on oxidative phosphorylation and $\beta$-fatty acid oxidation may be more vulnerable to oxidative stress both in utero and after birth.

\section{Role of Reactive Oxygen Molecules in Epigenesis}

Oxidant molecules can directly interact with DNA base pairs causing both genetic, as well as, epigenetic changes, the latter through alterations in DNA methylation and histone modification [61]. The cellular redox status influences gene expression and cell differentiation [23, 61]. The influence of ROS on epigenetic alterations in DNA methylation has been extensively studied in cancer [59]. Recently, the influence of ROS on DNA methylation is considered an important process of altered gene expression [59, 61]. Epigenesis is a process by which gene expression is either suppressed or enhanced without changes in primary DNA sequences but rather changes in the capacity of transcriptional control regions to induce gene expression [59].

DNA methylation is a prominent modification that leads to gene suppression of mRNA transcription and protein synthesis [59]. DNA methyltransferases methylate CpG islands, which influence the binding of transcription factors and/or their coregulators. Besides serving as signaling factors in transcription, ROS can interact directly with DNA resulting in oxidative damage and DNA breaks. Further, oxidant-mediated DNA breaks provide access to sites for DNA methyltransferases, which promote DNA methylation.

Modification of gene expression can also occur by altering the regulatory roles of histones. Chromatin is DNA wound around histones and gene expression is regulated by allowing access of transcription factors and RNA polymerase to DNA binding sites. Aminoterminal tails of histones are susceptible to posttranslational modification, such as, methylation, acetylation, phosphorylation, and ubiquitination [59]. Besides acting as signaling molecules in modifying histone function, ROS can also directly interact with histones resulting in disruption of normal gene expression.

\section{Oxidative Stress and Fetal Programming}

Fetal origins of adult disease are associated with several causative mechanisms depending on the conditions of intrauterine stress $[6,54,62]$. The role of oxidative stress in fetal programming is supported by epidemiological evidence of oxidant indices and low birth weight in association with type 2 diabetes [63], cardiovascular disease [64], and preeclampsia [28]. Thus, oxidative stress may be a connecting link between intrauterine insult and programming consequences after birth.

Children (8-13 years old) born growth restricted exhibit increased levels of lipid peroxidation and have higher blood pressures compared to age-matched children of normal birth weight [65-67]. Intrauterine growth-restricted (IUGR) infants had increased serum levels of both oxidant (lipid peroxidation and DNA damage) and antioxidant indices (superoxide dismutase, catalase, glutathione peroxidase) [68]. Indices of lipid peroxidation were elevated in placenta of women with preeclampsia $[69,70]$ and in plasma or serum of women with IUGR fetuses [71-73]. Oxidative stress associated with pregnancy complications may be a contributing factor in postnatal consequences of the neonate.

Animal studies support the hypothesis that oxidative stress induces programmed phenotypes in the adult offspring. Pregnant rats fed a low-protein diet have offspring (10-12 weeks old) that exhibit elevated arterial blood pressures and increased vasoconstrictor responsiveness to angiotensin II [74]. Prenatal treatment with the antioxidant agent, lazaroid, prevented the alterations in the cardiovascular responses associated with maternal protein restriction. In a separate study, maternal low-protein diet impaired the recovery to ischemia/reperfusion injury of offspring rat hearts, which was reduced with postnatal administration of the antioxidant, $\mathrm{N}$-acetylcysteine [75]. Maternal administration of the steroid, dexamethasone, generates offspring that exhibit increased ROS production (i.e., $\mathrm{H}_{2} \mathrm{O}_{2}$ ) in the coronary circulation [76] and in mitochondria of hearts of programmed sheep [77]. Postnatal glucocorticoid administration to 1-6-day-old rat pups increased vasoconstrictor responsiveness to $\mathrm{KCl}$ and the thromboxane analog, U46619, which was restored to normal function with Vitamin C and E treatment [78] suggesting that excessive glucocorticoid exposure promotes oxidative stress.

Prenatal hypoxia has been shown to generate oxidative stress in fetal hearts in a variety of animal species, such as, sheep [79], rat [36, 41], and guinea pig [42-44]. Maternal hypoxia increases expression levels of fetal cardiac inducible NO synthase $[42,44]$, nitrotyrosine $[36,43]$, heat shock protein 70 (HSP70) [36, 80], proinflammatory cytokines [81], and matrix metalloproteinases [44, 81-83] suggesting the generation of oxidative and inflammatory stress as causative. Prenatal treatment with the antioxidant, $\mathrm{N}$-acetylcysteine, inhibited the hypoxia-induced increase in peroxynitrite levels and fibrosis in fetal guinea pig heart ventricles [43]. 
Prenatal vitamin $\mathrm{C}$ inhibited the hypoxia-induced increase in fetal cardiac HSP70 expression and adult myocardial contractility associated with $\beta 1$ adrenoceptor stimulation in rat offspring [36]. With prenatal hypoxia, hearts of 6 month old male rat offspring exhibit reduced recovery to ischemia/reperfusion injury [80] in a sex-dependent manner with males more vulnerable than females [84] suggesting a protective influence of estrogen. In addition, exposure to prenatal hypoxia reduces cardiac efficiency of isolated perfused hearts of 4- and 12-month-old rat offspring during reperfusion following an ischemic period. The prenatal hypoxic insult causes growth restriction of the offspring and alters cardiac performance attributed to reduced glucose oxidation [49]. Furthermore, hyperoxic exposure of neonatal rats increases ROS production and is associated with increased systolic blood pressure and vascular dysfunction in adults [85]. Taken together, these studies suggest that oxidative stress is an important stressor that impacts the fetal heart and initiates cardiovascular programming of the offspring.

Prenatal hypoxia has also been shown to alter vascular reactivity of the adult offspring [51-53]. Myogenic response of mesenteric arteries from male 7-month-old rats exposed to prenatal hypoxia was greater compared to their normoxic controls [53]. This was attributed to reduced $\mathrm{NO}$ and vasodilator prostaglandin bioavailability in the offspring arteries. In separate studies, isolated mesenteric arteries of 4- and 12-month old rats exposed to hypoxia in utero exhibited decreased flow-mediated vasodilation in a pressure myograph [51] and endothelium-dependent relaxation to methacholine stimulation in a wire myograph [52]. A hypoxia-induced decrease in dilator responsiveness was associated with a decrease in $\mathrm{NO}$-dependent relaxation in the offspring that was attributed to mechanisms associated with a premature aging process. The role of oxidative stress was not investigated.

In addition, other organs are affected by oxidative stress during fetal development. Reduced placental perfusion is associated with oxidative stress in IUGR fetuses [72, 86-89]. Pancreatic $\beta$ cells of offspring of IUGR fetuses are vulnerable to oxidative stress because of a low-antioxidant capacity and exhibit increased ROS production and impaired ATP generation [90]. Prenatal hypoxia increased DNA fragmentation and lipid peroxidation in fetal guinea pig livers, which was reversed by the antioxidant, $\mathrm{N}$-acetylcysteine [46]. In livers of IUGR offspring, there is an upregulation of MnSOD as a compensatory adaptation that counters the decrease in pyruvate oxidation [91]. Prenatal hypoxia decreases insulinsignaling proteins in livers but not skeletal muscle of rat offspring [47]. Oxidative stress in hepatic tissue renders the adult liver susceptible to nonalcohol-associated fatty liver disease [92]. The kidney also plays an important role in programmed hypertension when exposed to an intrauterine environment that alters oxidative stress pathways. A homozygous NO synthase 3 deficient mouse was cross bred to generate paternally and maternally derived heterozygous offspring with high blood pressures [93]. Offspring that developed in a high-oxidative stress environment exhibited increased expression of peroxiredoxin, HSPB6, SOD-1, and
PPAR $\gamma$ in kidney but not liver tissue, compared to their counterparts who developed in a normal environment, identifying a role of oxidative stress in contributing to developmental programming of kidney-associated hypertension. Overall, reduced fetal oxygenation may impart organ-specific dysfunction via oxidative stress.

\section{Oxidative Stress and Epigenetic Mechanisms of Fetal Programming}

Animal studies have generated strong support for the role of epigenesis in response to altered maternal nutrition as an important programming mechanism in the offspring [94-96]. Both over- and undernutrition have been reported to induce DNA methylation of selected gene promoter regions [94-96]. Oxidative stress has also been identified as a contributing factor in epigenetic mechanisms [23, 61, 95, 96]. The role of oxidative stress in contributing to epigenetic modifications, independent of nutritional effects, warrants further study. The challenge is to link oxidative stress during pregnancy to specific target sites of epigenetic change in the offspring. Because ROS interacts promiscuously, we are limited in our ability to identify ROS-specific signaling within the cell [97].

Despite these limitations, intrauterine hypoxia and IUGR have been shown to alter DNA methylation of selected genes in several tissues including placenta [98], heart [99, 100], pancreas [101], liver [95], adrenal gland [102] and pulmonary arteries [103]. The following studies have identified specific target genes in fetal and offspring organs whose expression is altered by changes in DNA methylation associated with intrauterine hypoxia, IUGR, and protein restriction.

Chronic fetal hypoxia has been shown to decrease protein kinase $\mathrm{C}$ epsilon (PKCe) mRNA and protein expression in fetal and adult rat hearts [84]. The hypoxia-induced decrease in $\mathrm{PKC} \varepsilon$ expression is associated with methylation of $\mathrm{CpG}$ sites, specific for two SP1 binding sites in the PKCE promoter region in both ex vivo treatment of fetal hearts and in $\mathrm{H} 9 \mathrm{c} 2$ cells, an isolated embryonic ventricular myocyte cell line [99]. The epigenetic modification by hypoxia was further demonstrated by restoring the decrease in PKC $\varepsilon$ mRNA and protein expression by inhibiting DNA methyltransferase I with 5-aza-2'-deoxycytodine. This study links the epigenetic modification of $\mathrm{PKC} \varepsilon$ expression in adult offspring hearts with prenatal exposure to chronic hypoxia.

In pulmonary arteries of offspring mice exposed to two weeks of hypoxia during pregnancy, DNA methylation was increased and was associated with reduced pulmonary artery endothelium-dependent vasodilation [103]. The hypoxiainduced decrease in dilator responses to acetylcholine was reversed in the presence of histone deacetylase inhibitors, buryrate, and trichostatin $\mathrm{A}$, in association with reduced DNA methylation.

The fetal pancreas is vulnerable to oxidative stress because of its reduced antioxidant capacity $[104,105]$. In IUGR, rat pancreas exhibited reduced gene expression of the transcription factor, PDX1, which regulates pancreatic 
development and $\beta$-cell differentiation [101]. Pancreatic $\beta$ cells of IUGR rat fetuses,exhibited reduced expression of $P d x 1$, a homeobox gene required for pancreatic development and insulin production, as a result of epigenetic modifications throughout development [106]. The inhibition of $P d x 1$ expression progressed in IUGR fetuses after the recruitment of histone deacetylase 1 to the $P d x 1$ promoter and subsequent demethylation of histones $\mathrm{H} 3$ and $\mathrm{H} 4$. In 2week-old and 6-month old islets [106], H3 was permanently methylated. It is proposed that progressive DNA methylation inhibits gene expression and may link gene silencing in the $\beta$ cells to the development of a diabetic phenotype [84]. Although not hypoxia-induced, protein restriction increases the expression of peroxisome-proliferator-activated receptor alpha (PPAR $\alpha$ ) protein and glucocorticoid receptors in the offspring rat liver and is associated with hypomethylation of their promoter regions [96]. In adrenal gland of rat offspring (4-week-old), maternal protein restriction increased expression of angiotensin receptor AT1b, which correlated with hypomethylation of its promoter and was reversed with maternal administration of $11 \beta$-hydroxylase inhibitor, metyrapone, suggesting that maternal glucocorticoid induces changes in methylation in fetal adrenal glands [102].

Further, excess glucocorticoid exposure during pregnancy was also shown to induce epigenetic responses contributing to programming effects in the offspring [107]. For example, glucocorticoid exposure of isolated cultured rat hepatocytes induced DNA demethylation of a specific gene locus (Tat) associated with a glucocorticoid response unit [108]. This demonstrated that glucocorticoid may regulate DNA demethylation as a mechanism of modulating gene expression to subsequent stimulation by glucocorticoid receptors. In embryonic cortical neural stem cells, glucocorticoid decreased global DNA methylation, which is retained in daughter cells [109]. These studies implicate glucocorticoid as activating sustained epigenetic modifications that may result in long-term consequences associated with specific cell-related phenotypes.

\section{Conclusions}

ROS generated by a variety of intrauterine conditions may be one of the key downstream mediators that initiates epigenesis and programming of the offspring. ROS generation normally is balanced by the cell's antioxidant defense mechanisms, which maintains its redox state and is important in physiological regulation in both the embryo and fetus. Prenatal hypoxia, nutritional deficiency/excess, and glucocorticoid exposure are each capable of generating excessive ROS levels by differing mechanisms. Organ-specific responses are dependent on the relative balance between ROS generation and the antioxidant capacity of the cell. Identifying the impact of oxidative stress on gene targets will be important for understanding the cell-specific responses to intrauterine stress, as well as, developing therapeutic strategies for alleviating long-term programmed consequences associated with adult disease.

\section{References}

[1] G. J. Burton, "Oxygen, the Janus gas; its effects on human placental development and function," Journal of Anatomy, vol. 215, no. 1, pp. 27-35, 2009.

[2] M. E. Symonds, T. Stephenson, D. S. Gardner, and H. Budge, "Long-term effects of nutritional programming of the embryo and fetus: mechanisms and critical windows," Reproduction, Fertility and Development, vol. 19, no. 1, pp. 53-63, 2007.

[3] E. A. Herrera, A. D. Kane, J. A. Hansell et al., "A role for xanthine oxidase in the control of fetal cardiovascular function in late gestation sheep," Journal of Physiology, vol. 590, no. 8, pp. 1825-1837, 2012.

[4] A. S. Thakor, H. G. Richter, A. D. Kane et al., "Redox modulation of the fetal cardiovascular defence to hypoxaemia," Journal of Physiology, vol. 588, no. 21, pp. 4235-4247, 2010.

[5] A. S. Thakor, E. A. Herrera, M. Serón-Ferré, and D. A. Giussani, "Melatonin and vitamin C increase umbilical blood flow via nitric oxide-dependent mechanisms," Journal of Pineal Research, vol. 49, no. 4, pp. 399-406, 2010.

[6] M. A. Hanson and P. D. Gluckman, "Developmental origins of health and disease: new insights," Basic and Clinical Pharmacology and Toxicology, vol. 102, no. 2, pp. 90-93, 2008.

[7] A. M. Nuyt and B. T. Alexander, "Developmental programming and hypertension," Current Opinion in Nephrology and Hypertension, vol. 18, no. 2, pp. 144-152, 2009.

[8] T. H. Nesterenko and H. Aly, "Fetal and neonatal programming: evidence and clinical implications," American Journal of Perinatology, vol. 26, no. 3, pp. 191-198, 2009.

[9] S. K. Barnes and S. E. Ozanne, "Pathways linking the early environment to long-term health and lifespan," Progress in Biophysics and Molecular Biology, vol. 106, no. 1, pp. 323-336, 2011.

[10] D. J. P. Barker, C. Osmond, J. Golding, D. Kuh, and M. E. J. Wadsworth, "Growth in utero, blood pressure in childhood and adult life, and mortality from cardiovascular disease," British Medical Journal, vol. 298, no. 6673, pp. 564-567, 1989.

[11] D. J. P. Barker, C. Osmond, S. J. Simmonds, and G. A. Wield, "The relation of small head circumference and thinness at birth to death from cardiovascular disease in adult life," British Medical Journal, vol. 306, no. 6875, pp. 422-426, 1993.

[12] C. Osmond, D. J. P. Barker, P. D. Winter, C. H. D. Fall, and S. J. Simmonds, "Early growth and death from cardiovascular disease in women," British Medical Journal, vol. 307, no. 6918, pp. 1519-1524, 1993.

[13] D. J. P. Barker, A. R. Bull, C. Osmond, and S. J. Simmonds, "Fetal and placental size and risk of hypertension in adult life," British Medical Journal, vol. 301, no. 6746, pp. 259-262, 1990.

[14] J. G. Eriksson, T. Forsén, J. Tuomilehto, V. W. V. Jaddoe, C. Osmond, and D. J. P. Barker, "Effects of size at birth and childhood growth on the insulin resistance syndrome in elderly individuals," Diabetologia, vol. 45, no. 3, pp. 342-348, 2002.

[15] J. G. Eriksson, T. J. Forsen, C. Osmond, and D. J. P. Barker, "Pathways of infant and childhood growth that lead to type 2 diabetes," Diabetes Care, vol. 26, no. 11, pp. 3006-3010, 2003.

[16] G. C. Curhan, W. C. Willett, E. B. Rimm, D. Spiegelman, A. L. Ascherio, and M. J. Stampfer, "Birth weight and adult hypertension, diabetes mellitus, and obesity in US men," Circulation, vol. 94, no. 12, pp. 3246-3250, 1996. 
[17] G. C. Curhan, G. M. Chertow, W. C. Willett et al., "Birth weight and adult hypertension and obesity in women," Circulation, vol. 94, no. 6, pp. 1310-1315, 1996.

[18] C. N. Hales and D. J. P. Barker, "The thrifty phenotype hypothesis," British Medical Bulletin, vol. 60, pp. 5-20, 2001.

[19] P. D. Gluckman, M. A. Hanson, and H. G. Spencer, "Predictive adaptive responses and human evolution," Trends in Ecology and Evolution, vol. 20, no. 10, pp. 527-533, 2005.

[20] W. W. Hay Jr., "Strategies for feeding the preterm infant," Neonatology, vol. 94, no. 4, pp. 245-254, 2008.

[21] D. J. P. Barker, C. Osmond, T. J. Forsén, E. Kajantie, and J. G. Eriksson, "Trajectories of growth among children who have coronary events as adults," The New England Journal of Medicine, vol. 353, no. 17, pp. 1802-1809, 2005.

[22] P. A. Dennery, "Oxidative stress in development: nature or nurture?" Free Radical Biology and Medicine, vol. 49, no. 7, pp. 1147-1151, 2010.

[23] M. J. Hitchler and F. E. Domann, "An epigenetic perspective on the free radical theory of development," Free Radical Biology and Medicine, vol. 43, no. 7, pp. 1023-1036, 2007.

[24] F. Q. Schafer and G. R. Buettner, "Redox environment of the cell as viewed through the redox state of the glutathione disulfide/glutathione couple," Free Radical Biology and Medicine, vol. 30, no. 11, pp. 1191-1212, 2001.

[25] V. Castagné, K. Lefèvre, R. Natero, D. A. Becker, and P. G. H. Clarke, "An optimal redox status for the survival of axotomized ganglion cells in the developing retina," Neuroscience, vol. 93, no. 1, pp. 313-320, 1999.

[26] D. A. Giussani, C. E. Salinas, M. Villena, and C. E. Blanco, "The role of oxygen in prenatal growth: studies in the chick embryo," Journal of Physiology, vol. 585, no. 3, pp. 911-917, 2007.

[27] L. Myatt, "Review: reactive oxygen and nitrogen species and functional adaptation of the placenta," Placenta, vol. 31, pp. S66-S69, 2010.

[28] J. M. Roberts and K. Y. Lain, "Recent insights into the pathogenesis of pre-eclampsia," Placenta, vol. 23, no. 5, pp. 359372, 2002.

[29] L. G. Moore, "Human genetic adaptation to high altitude," High Altitude Medicine and Biology, vol. 2, no. 2, pp. 257-279, 2001.

[30] D. A. Giussani, P. S. Phillips, S. Anstee, and D. J. P. Barker, "Effects of altitude versus economic status on birth weight and body shape at birth," Pediatric Research, vol. 49, no. 4, pp. 490-494, 2001.

[31] S. Niermeyer, P. A. Mollinedo, and L. Huicho, "Child health and living at high altitude," Archives of Disease in Childhood, vol. 94, no. 10, pp. 806-811, 2009.

[32] C. G. Julian, M. J. Wilson, and L. G. Moore, "Evolutionary adaptation to high altitude: a view from in utero," American Journal of Human Biology, vol. 21, no. 5, pp. 614-622, 2009.

[33] S. Zamudio, "High-altitude hypoxia and preeclampsia," Frontiers in Bioscience, vol. 12, no. 8, pp. 2967-2977, 2007.

[34] Y. Li, P. Gonzalez, and L. Zhang, "Fetal stress and programming of hypoxic/ischemic-sensitive phenotype in the neonatal brain: mechanisms and possible interventions," Progress in Neurobiology, vol. 98, no. 2, pp. 145-165, 2012.

[35] E. J. Camm, J. A. Hansell, A. D. Kane et al., "Partial contributions of developmental hypoxia and undernutrition to prenatal alterations in somatic growth and cardiovascular structure and function," American Journal of Obstetrics and Gynecology, vol. 203, no. 5, pp. 495.e24-495.e34, 2010.
[36] D. A. Giussani, E. J. Camm, Y. Niu et al., "Developmental programming of cardiovascular dysfunction by prenatal hypoxia and oxidative stress," PLoS ONE, vol. 7, no. 2, Article ID e31017, 2012.

[37] J. L. Morrison, "Sheep models of intrauterine growth restriction: fetal adaptations and consequences," Clinical and Experimental Pharmacology and Physiology, vol. 35, no. 7, pp. 730-743, 2008.

[38] Y. Dong, Z. Yu, Y. Sun et al., "Chronic fetal hypoxia produces selective brain injury associated with altered nitric oxide synthases," American Journal of Obstetrics and Gynecology, vol. 204, no. 3, pp. 254e16-254e28, 2011.

[39] R. Guo, W. Hou, Y. Dong, Z. Yu, J. Stites, and C. P. Weiner, "Brain injury caused by chronic fetal hypoxemia is mediated by inflammatory cascade activation," Reproductive Sciences, vol. 17, no. 6, pp. 540-548, 2010.

[40] A. J. Gunn and L. Bennet, "Fetal hypoxia insults and patterns of brain injury: insights from animal models," Clinics in Perinatology, vol. 36, no. 3, pp. 579-593, 2009.

[41] L. Zhang, "Prenatal hypoxia and cardiac programming," Journal of the Society for Gynecologic Investigation, vol. 12, no. 1, pp. 2-13, 2005.

[42] L. P. Thompson and Y. Dong, "Chronic hypoxia decreases endothelial nitric oxide synthase protein expression in fetal guinea pig hearts," Journal of the Society for Gynecologic Investigation, vol. 12, no. 6, pp. 388-395, 2005.

[43] L. C. Evans, H. Liu, G. A. Pinkas, and L. P. Thompson, "Chronic hypoxia increases peroxynitrite, MMP9 expression, and collagen accumulation in fetal guinea pig hearts," Pediatric Research, vol. 71, no. 1, pp. 25-31, 2012.

[44] Y. Dong and L. P. Thompson, "Differential expression of endothelial nitric oxide synthase in coronary and cardiac tissue in hypoxic fetal guinea pig hearts," Journal of the Society for Gynecologic Investigation, vol. 13, no. 7, pp. 483-490, 2006.

[45] K. Hashimoto, G. Pinkas, L. Evans, H. Liu, Y. Al-Hasan, and L. P. Thompson, "Protective effect of N-acetylcysteine on liver damage during chronic intrauterine hypoxia in fetal guinea pig ," Reproductive Sciences. In press.

[46] C. F. Rueda-Clausen, V. W. Dolinsky, J. S. Morton, S. D. Proctor, J. R. B. Dyck, and S. T. Davidge, "Hypoxia-induced intrauterine growth restriction increases the susceptibility of rats to high-fat diet-induced metabolic syndrome," Diabetes, vol. 60, no. 2, pp. 507-516, 2011.

[47] E. J. Camm, M. S. Martin-Gronert, N. L. Wright, J. A. Hansell, S. E. Ozanne, and D. A. Giussani, "Prenatal hypoxia independent of undernutrition promotes molecular markers of insulin resistance in adult offspring," FASEB Journal, vol. 25, no. 1, pp. 420-427, 2011.

[48] C. Mao, J. Hou, J. Ge et al., "Changes of renal $\mathrm{AT}_{1} / \mathrm{AT}_{2}$ receptors and structures in ovine fetuses following exposure to long-term hypoxia," American Journal of Nephrology, vol. 31, no. 2, pp. 141-150, 2010.

[49] C. F. Rueda-Clausen, J. S. Morton, G. D. Lopaschuk, and S. T. Davidge, "Long-term effects of intrauterine growth restriction on cardiac metabolism and susceptibility to ischaemia/reperfusion," Cardiovascular Research, vol. 90, no. 2, pp. 285-294, 2011.

[50] C. F. Rueda-Clausen, J. S. Morton, and S. T. Davidge, "Effects of hypoxia-induced intrauterine growth restriction on cardiopulmonary structure and function during adulthood," Cardiovascular Research, vol. 81, no. 4, pp. 713-722, 2009.

[51] J. S. Morton, C. F. Rueda-Clausen, and S. T. Davidge, "Flowmediated vasodilation is impaired in adult rat offspring 
exposed to prenatal hypoxia," Journal of Applied Physiology, vol. 110, no. 4, pp. 1073-1082, 2011.

[52] J. S. Morton, C. F. Rueda-Clausen, and S. T. Davidge, "Mechanisms of endothelium-dependent vasodilation in male and female, young and aged offspring born growth restricted," American Journal of Physiology, vol. 298, no. 4, pp. R930R938, 2010.

[53] D. G. Hemmings, S. J. Williams, and S. T. Davidge, "Increased myogenic tone in 7-month-old adult male but not female offspring from rat dams exposed to hypoxia during pregnancy," American Journal of Physiology, vol. 289, no. 2, pp. H674H682, 2005.

[54] Z. C. Luo, W. D. Fraser, P. Julien et al., "Tracing the origins of "fetal origins" of adult diseases: programming by oxidative stress?" Medical Hypotheses, vol. 66, no. 1, pp. 38-44, 2006.

[55] D. Shao, S. I. Oka, C. D. Brady, J. Haendeler, P. Eaton, and J. Sadoshima, "Redox modification of cell signaling in the cardiovascular system," Journal of Molecular and Cellular Cardiology, vol. 52, no. 3, pp. 550-558, 2012.

[56] S. Cadenas, J. Aragonés, and M. O. Landázuri, "Mitochondrial reprogramming through cardiac oxygen sensors in ischaemic heart disease," Cardiovascular Research, vol. 88, no. 2, pp. 219-228, 2010.

[57] G. L. Semenza, "Oxygen-dependent regulation of mitochondrial respiration by hypoxia-inducible factor 1," Biochemical Journal, vol. 405, no. 1, pp. 1-9, 2007.

[58] W. Droge, "Free radicals in the physiological control of cell function," Physiological Reviews, vol. 82, pp. 47-95, 2002.

[59] D. Ziech, R. Franco, A. Pappa, and M. I. Panayiotidis, "Reactive Oxygen Species (ROS)-Induced genetic and epigenetic alterations in human carcinogenesis," Mutation Research, vol. 711, no. 1-2, pp. 167-173, 2011.

[60] M. A. Aon, S. Cortassa, F. G. Akar, and B. O'Rourke, "Mitochondrial criticality: a new concept at the turning point of life or death," Biochimica et Biophysica Acta, vol. 1762, no. 2, pp. 232-240, 2006.

[61] S. Cerda and S. A. Weitzman, "Influence of oxygen radical injury on DNA methylation," Mutation Research, vol. 386, no. 2, pp. 141-152, 1997.

[62] A. Singhal and A. Lucas, "Early origins of cardiovascular disease: is there a unifying hypothesis?" The Lancet, vol. 363, no. 9421, pp. 1642-1645, 2004.

[63] E. Peuchant, J. L. Brun, V. Rigalleau et al., "Oxidative and antioxidative status in pregnant women with either gestational or type 1 diabetes," Clinical Biochemistry, vol. 37, no. 4, pp. 293-298, 2004.

[64] G. Bloc, M. Dietrich, E. P. Norkus et al., "Factors associated with oxidative stress in human populations," American Journal of Epidemiology, vol. 156, pp. 274-285, 2002.

[65] A. Mohn, V. Chiavaroli, M. Cerruto et al., "Increased oxidative stress in prepubertal children born small for gestational age," Journal of Clinical Endocrinology and Metabolism, vol. 92, no. 4, pp. 1372-1378, 2007.

[66] M. C. P. Franco, E. M. Kawamoto, R. Gorjão et al., "Biomarkers of oxidative stress and antioxidant status in children born small for gestational age: evidence of lipid peroxidation," Pediatric Research, vol. 62, no. 2, pp. 204-208, 2007.

[67] V. Chiavaroli, C. Giannini, E. D’Adamo, T. De Giorgis, F. Chiarelli, and A. Mohn, "Insulin resistance and oxidative stress in children born small and large for gestational age," Pediatrics, vol. 124, no. 2, pp. 695-702, 2009.
[68] Z. Hracsko, H. Orvos, Z. Novak, A. Pal, and I. S. Varga, "Evaluation of oxidative stress markers in neonates with intra-uterine growth retardation," Redox Report, vol. 13, no. 1, pp. 11-16, 2008.

[69] G. Bayhan, Y. Koçyigit, A. Atamer, Y. Atamer, and Z. Akkus, "Potential atherogenic roles of lipids, lipoprotein(a) and lipid peroxidation in preeclampsia," Gynecological Endocrinology, vol. 21, no. 1, pp. 1-6, 2005.

[70] Y. Atamer, Y. Koçyigit, B. Yokus, A. Atamer, and A. C. Erden, "Lipid peroxidation, antioxidant defense, status of trace metals and leptin levels in preeclampsia," European Journal of Obstetrics Gynecology and Reproductive Biology, vol. 119, no. 1, pp. 60-66, 2005.

[71] A. Karowicz-Bilińska, U. Kowalska-Koprek, J. Suzin, and P. Sieroszewski, "Analysis of 8-isoprostane concentration as a marker of oxidative stress in pregnant women diagnosed with IUGR," Ginekologia Polska, vol. 74, no. 10, pp. 1137-1142, 2003.

[72] A. Karowicz-Bilinska, K. Kędziora-Kornatowska, and G. Bartosz, "Indices of oxidative stress in pregnancy with fetal growth restriction," Free Radical Research, vol. 41, no. 8, pp. 870-873, 2007.

[73] A. Biri, N. Bozkurt, A. Turp, M. Kavutcu, Ö. Himmetoglu, and I. Durak, "Role of oxidative stress in intrauterine growth restriction," Gynecologic and Obstetric Investigation, vol. 64, no. 4, pp. 187-192, 2007.

[74] G. Cambonie, B. Comte, C. Yzydorczyk et al., "Antenatal antioxidant prevents adult hypertension, vascular dysfunction, and microvascular rarefaction associated with in utero exposure to a low-protein diet," American Journal of Physiology, vol. 292, no. 3, pp. R1236-R1245, 2007.

[75] M. J. Elmes, D. S. Gardner, and S. C. Langley-Evans, "Fetal exposure to a maternal low-protein diet is associated with altered left ventricular pressure response to ischaemiareperfusion injury," British Journal of Nutrition, vol. 98, no. 1, pp. 93-100, 2007.

[76] R. D. Roghair, F. J. Miller, T. D. Scholz, F. S. Lamb, and J. L. Segar, "Endothelial superoxide production is altered in sheep programmed by early gestation dexamethasone exposure," Neonatology, vol. 93, no. 1, pp. 19-27, 2007.

[77] N. H. Von Bergen, S. L. Koppenhafer, D. R. Spitz et al., "Fetal programming alters reactive oxygen species production in sheep cardiac mitochondria," Clinical Science, vol. 116, no. 8, pp. 659-668, 2009.

[78] E. A. Herrera, M. M. Verkerk, J. B. Derks, and D. A. Giussani, "Antioxidant treatment alters peripheral vascular dysfunction induced by postnatal glucocorticoid therapy in rats," PLoS ONE, vol. 5, no. 2, Article ID e9250, 2010.

[79] J. B. Derks, M. A. Oudijk, H. L. Torrance et al., "Allopurinol reduces oxidative stress in the ovine fetal cardiovascular system after repeated episodes of ischemia-reperfusion," Pediatric Research, vol. 68, no. 5, pp. 374-380, 2010.

[80] G. Li, Y. Xiao, J. L. Estrella, C. A. Ducsay, R. D. Gilbert, and L. Zhang, "Effect of fetal hypoxia on heart susceptibility to ischemia and reperfusion injury in the adult rat," Journal of the Society for Gynecologic Investigation, vol. 10, no. 5, pp. 265-274, 2003.

[81] C. Oh, Y. Dong, H. Liu, and L. P. Thompson, "Intrauterine hypoxia upregulates proinflammatory cytokines and matrix metalloproteinases in fetal guinea pig hearts," American Journal of Obstetrics and Gynecology, vol. 199, no. 1, pp. 78.e1-78.e6, 2008. 
[82] W. Tong and L. Zhang, "Fetal hypoxia and programming of matrix metalloproteinases," Drug Discovery Today, vol. 17, pp. 124-134, 2012.

[83] W. Tong, Q. Xue, Y. Li, and L. Zhang, "Maternal hypoxia alters matrix metalloproteinase expression patterns and causes cardiac remodeling in fetal and neonatal rats," American Journal of Physiology, vol. 301, no. 5, pp. H2113-H21121, 2011.

[84] Q. Xue and L. Zhang, "Prenatal hypoxia causes a sexdependent increase in heart susceptibility to ischemia and reperfusion injury in adult male offspring: role of protein kinase Ce," Journal of Pharmacology and Experimental Therapeutics, vol. 330, no. 2, pp. 624-632, 2009.

[85] C. Yzydorczyk, B. Comte, G. Cambonie et al., "Neonatal oxygen exposure in rats leads to cardiovascular and renal alterations in adulthood," Hypertension, vol. 52, no. 5, pp. 889-895, 2008.

[86] H. G. Richter, E. J. Camm, B. N. Modi et al., "Ascorbate prevents placental oxidative stress and enhances birth weight in hypoxic pregnancy in rats," Journal of Physiology, vol. 590, no. 6, pp. 1377-1387, 2012.

[87] N. Potdar, R. Singh, V. Mistry et al., "First-trimester increase in oxidative stress and risk of small-for-gestational-age fetus," An International Journal of Obstetrics and Gynaecology, vol. 116, no. 5, pp. 637-642, 2009.

[88] G. J. Burton, H. W. Yung, T. Cindrova-Davies, and D. S. Charnock-Jones, "Placental endoplasmic reticulum stress and oxidative stress in the pathophysiology of unexplained intrauterine growth restriction and early onset preeclampsia," Placenta, vol. 30, pp. 43-48, 2009.

[89] A. Casasco, A. Calligaro, M. Casasco et al., "Immunohistochemical localization of lipoperoxidation products in normal human placenta," Placenta, vol. 18, no. 4, pp. 249-253, 1997.

[90] R. A. Simmons, I. Suponitsky-Kroyter, and M. A. Selak, "Progressive accumulation of mitochondrial DNA mutations and decline in mitochondrial function lead to $\beta$-cell failure," Journal of Biological Chemistry, vol. 280, no. 31, pp. 2878528791, 2005.

[91] I. E. Peterside, M. A. Selak, and R. A. Simmons, "Impaired oxidative phosphorylation in hepatic mitochondria in growth-retarded rats," American Journal of Physiology, vol. 285, no. 6, pp. E1258-E1266, 2003.

[92] G. H. Koek, P. R. Liedorp, and A. Bast, "The role of oxidative stress in non-alcoholic steatohepatitis," Clinica Chimica Acta, vol. 412, no. 15-16, pp. 1297-1305, 2011.

[93] L. M. Ghulmiyyah, M. M. Costantine, H. Yin et al., "The role of oxidative stress in the developmental origin of adult hypertension," American Journal of Obstetrics and Gynecology, vol. 205, no. 2, pp. 155.e7-155.e11, 2011.

[94] J. C. Mathers and J. A. McKay, "Epigenetics-potential contribution to fetal programming," in Early Nutrition Programming and Health Outcomes in Later Life: Obesity and Beyond, B. Koletzko et al., Ed., pp. 119-123, Springer Science +Business Media, 2009.

[95] W. D. Rees, H. H. Susan, D. S. Brown, C. Antipatis, and R. M. Palmer, "Maternal protein deficiency causes hypermethylation of DNA in the livers of rat fetuses," Journal of Nutrition, vol. 130, no. 7, pp. 1821-1826, 2000.

[96] K. A. Lillycrop, E. S. Phillips, C. Torrens, M. A. Hanson, A. A. Jackson, and G. C. Burdge, "Feeding pregnant rats a proteinrestricted diet persistently alters the methylation of specific cytosines in the hepatic PPAR $\alpha$ promoter of the offspring," British Journal of Nutrition, vol. 100, no. 2, pp. 278-282, 2008.
[97] T. Finkel, "Signal transduction by reactive oxygen species," Journal of Cell Biology, vol. 194, no. 1, pp. 7-15, 2011.

[98] C. P. Gheorghe, R. Goyal, A. Mittal, and L. D. Longo, "Gene expression in the placenta: maternal stress and epigenetic responses," International Journal of Developmental Biology, vol. 54, no. 2-3, pp. 507-523, 2010.

[99] A. J. Patterson, M. Chen, Q. Xue, D. Xiao, and L. Zhang, "Chronic prenatal hypoxia induces epigenetic programming of $\mathrm{PKC \varepsilon}$ gene repression in rat hearts," Circulation Research, vol. 107, no. 3, pp. 365-373, 2010.

[100] A. J. Patterson, D. Xiao, F. Xiong, B. Dixon, and L. Zhang, "Hypoxia-derived oxidative stress mediates epigenetic repression of $\mathrm{PKC} \varepsilon$ gene in foetal rat hearts," Cardiovascular Research, vol. 93, no. 2, pp. 302-310, 2012.

[101] S. E. Pinney and R. A. Simmons, "Metabolic programming, epigenetics, and gestational diabetes mellitus," Current Diabetes Reports, vol. 12, pp. 67-74, 2012.

[102] I. Bogdarina, A. Haase, S. Langley-Evans, and A. J. L. Clark, "Glucocorticoid effects on the programming of AT1b angiotensin receptor gene methylation and expression in the rat," PLoS ONE, vol. 5, no. 2, Article ID e9237, 2010.

[103] E. Rexhaj, J. Bloch, P. Y. Jayet et al., "Fetal programming of pulmonary vascular dysfunction in mice: role of epigenetic mechanisms," American Journal of Physiology, vol. 301, no. 1, pp. H247-H252, 2011.

[104] S. Lenzen, J. Drinkgern, and M. Tiedge, "Low antioxidant enzyme gene expression in pancreatic islets compared with various other mouse tissues," Free Radical Biology and Medicine, vol. 20, no. 3, pp. 463-466, 1996.

[105] M. Tiedge, S. Lortz, J. Drinkgern, and S. Lenzen, "Relation between antioxidant enzyme gene expression and antioxidative defense status of insulin-producing cells," Diabetes, vol. 46, no. 11, pp. 1733-1742, 1997.

[106] J. H. Park, D. A. Stoffers, R. D. Nicholls, and R. A. Simmons, "Development of type 2 diabetes following intrauterine growth retardation in rats is associated with progressive epigenetic silencing of Pdx1," Journal of Clinical Investigation, vol. 118, no. 6, pp. 2316-2324, 2008.

[107] J. R. Seckl, "Prenatal glucocorticoids and long-term programming," European Journal of Endocrinology, Supplement, vol. 151, no. 3, pp. U49-U62, 2004.

[108] H. Thomassin, M. Flavin, M. L. Espinás, and T. Grange, "Glucocorticoid-induced DNA demethylation and gene memory during development," EMBO Journal, vol. 20, no. 8, pp. 1974-1983, 2001.

[109] R. Bose, M. Moors, R. Tofighi, A. Cascante, O. Hermanson, and S. Ceccatelli, "Glucocorticoids induce long-lasting effects in neural stem cells resulting in senescence-related alterations," Cell Death and Disease, vol. 1, no. 11, article e92, 2010 . 


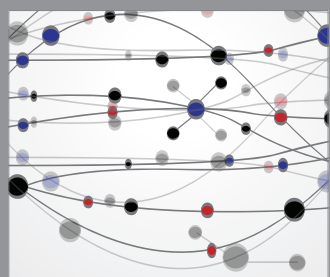

The Scientific World Journal
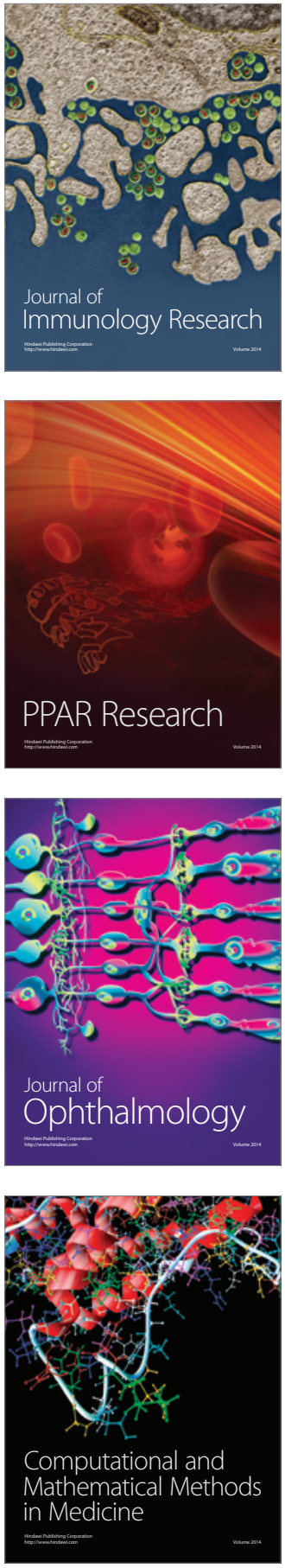

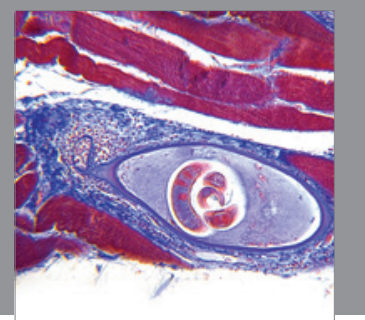

Gastroenterology

Research and Practice
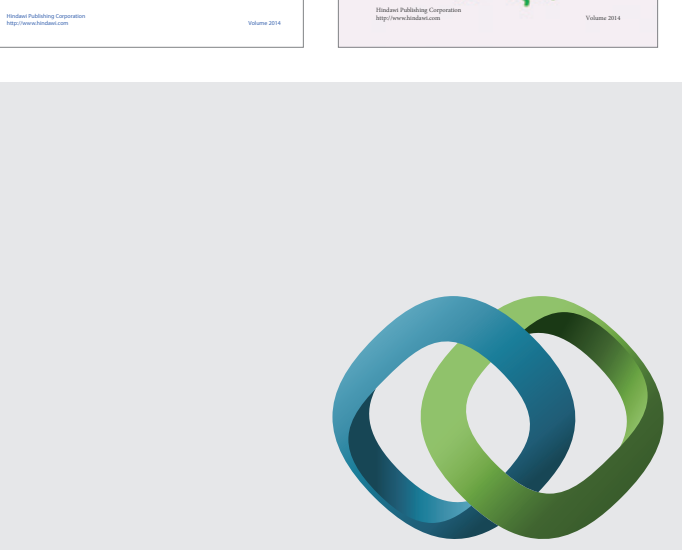

\section{Hindawi}

Submit your manuscripts at

http://www.hindawi.com
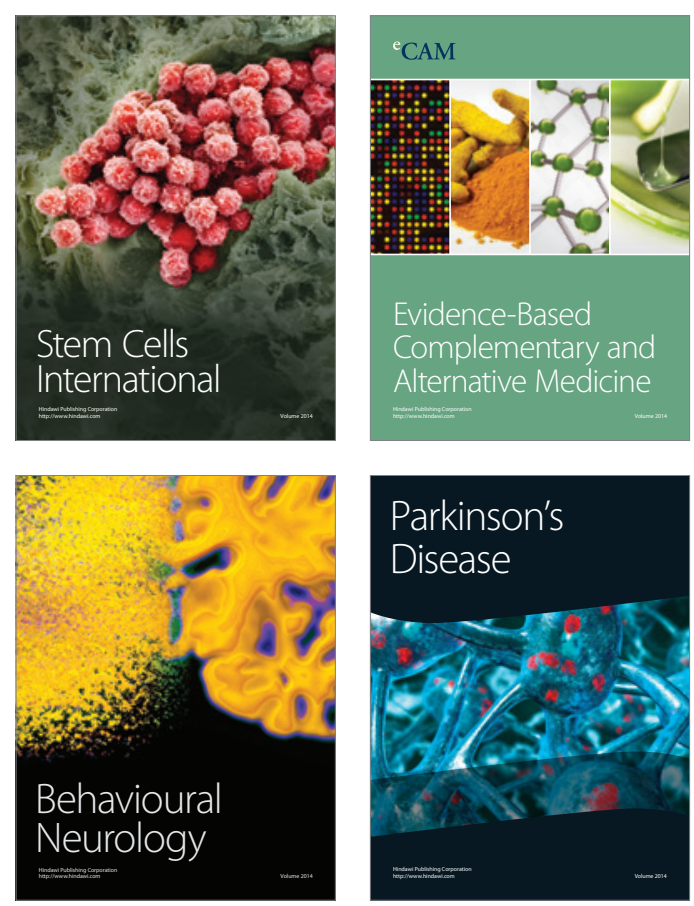

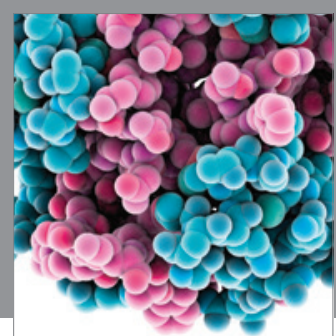

Journal of
Diabetes Research

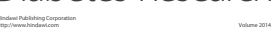

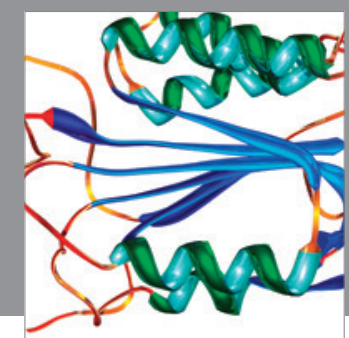

Disease Markers
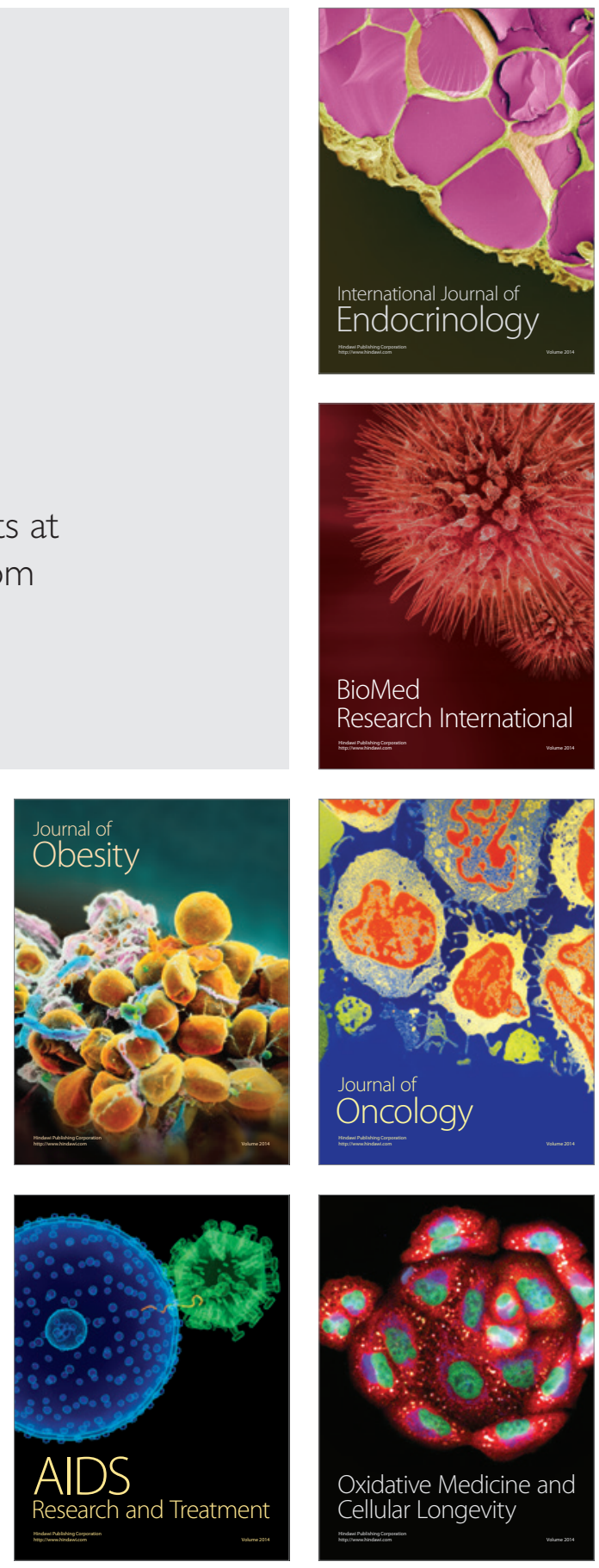